\title{
Clinical Relationship of Degenerative Changes between the Cervical and Lumbar Spine
}

\author{
Yuichiro Morishita ${ }^{1,2}$, Zorica Buser ${ }^{1}$, Anthony D’Oro ${ }^{1}$, Keiichiro Shiba ${ }^{2}$, Jeffrey C. Wang ${ }^{1}$ \\ ${ }^{1}$ Department of Orthopedic Surgery, Keck School of Medicine, University of Southern California, Los Angeles, CA, USA \\ ${ }^{2}$ Department of Orthopedic Surgery, Spinal Injuries Center, Iizuka, Japan
}

Study Design: Retrospective, observational, case series.

Purpose: To elucidate the prevalence of degenerative changes in the cervical and lumbar spine and estimate the degenerative changes in the cervical spine based on the degeneration of lumbar disc through a retrospective review of magnetic resonance (MR) images.

Overview of Literature: Over 50\% of middle-aged adults show evidence of spinal degeneration. However, the relationship between degenerative changes in the cervical and lumbar spine has yet to be elucidated.

Methods: A retrospective review of positional MR images of 152 patients with symptoms related to cervical and lumbar spondylosis with or without a neurogenic component was conducted. The degree of intervertebral disc degeneration (IDD) was assessed on a grade of 1-5 for each segment of the cervical and lumbar spine using MR T2-weighted sagittal images. The grades across all segments were summed to produce the degenerative disc score (DDS) for the cervical and lumbar spine. The patients were divided into two groups based on the IDD grade for each lumbar segment: normal (grades 1 and 2) and degenerative (grades 3-5).

Results: DDSs for the cervical and lumbar spine were positively correlated. Significant differences in cervical DDSs between the groups were observed in all lumbar segments. Although there were no significant differences in cervical DDSs among the degenerative lumbar segment, cervical DDSs at the L1-2 and L2-3 segments tended to be higher than those at the L3-4, L4-5, and L5-S degenerative segments.

Conclusions: Our study shows that participants with degenerative changes in the upper lumbar segments are more likely to have a certain amount of cervical spondylosis. This information could be used to lower the incidence of a missed diagnosis of cervical spine disorders in patients presenting with lumbar spine symptomology.

Keywords: Cervical spine; Lumbar spine; Positional magnetic resonance imaging; Intervertebral disc degeneration; Degenerative disc score

\section{Introduction}

Degenerative skeletal disorders are common and serious problems worldwide, particularly in the aging populations. These disorders are classified as polygenic diseases because they are developed as a consequence of the interaction between an individual's genetic, lifestyle, and environmental factors. Degenerative disease of the spine is a common manifestation of the degenerative process, with the earliest spinal lesions thought to occur in the in-

Received Jun 2, 2017; Revised Jul 28, 2017; Accepted Aug 7, 2017

Corresponding author: Zorica Buser

Department of Orthopaedic Surgery, Keck School of Medicine, University of Southern California, Hoffman Medical Research Center, 1450 Biggy Street, NRT-2509N, Los Angeles, CA 90033, USA

Tel: +1-323-442-0206, E-mail: zbuser@usc.edu 
tervertebral discs. Intervertebral disc degeneration (IDD) typically appears in the second decade of life in men and in the third decade in women, with more than 50\% of middle-aged adults showing some evidence of spinal spondylosis [1].

The human spine is subjected to large compressive preloads during activities of daily living. Depending on the position of the spine, the axial load exerted on the lumbar intervertebral discs can increase to magnitudes of up to three times the body weight. Age, individual somatotype, and environmental factors, such as activities of daily living and occupation, are the most important etiological factors of IDD [2]. Although not completely understood, genetic factors are increasingly being considered in the etiology of IDD. There is emerging evidence that gene polymorphisms in disc components, such as proteins, proteoglycans, cytokines, enzymes, and vitamin D receptor, play significant roles in the pathology of IDD, possibly by altering the normal homeostasis of the intervertebral disc and/or influencing the effects of environmental factors [3-7].

Although IDD is common, clinical diagnosis and treatments are complicated because patients frequently present with complex neurogenic patterns of pain and impairment related to degenerative changes at multiple spinal levels. In these cases, assessment and treatment are often focused on the spinal region associated with the patient's primary complaint. For instance, a clinician may associate complaints of gait disturbances reported by patients with radiological evidence of lumbar spinal canal stenosis to cauda equina syndrome, focusing the neurological examination on the lower extremities, such as assessment of deep tendon reflexes, without considering the contribution of upper spinal lesions. An issue limiting the assessment and treatment in these cases is the absence of information regarding the relationship between degenerative changes at different levels of the spine.

Numerous studies investigating the degenerative processes of the human spine have reported the results of diagnostic imaging for the assessment and identification of spinal degeneration, as well as the resulting effects of the degeneration on the kinematics of the spine [8-17]. However, to the best of our knowledge, few reports have so far investigated the correlation between degenerative changes of the cervical and lumbar spine. The aim of our study was to elucidate the prevalence of degenerative changes in the cervical and lumbar spine and estimate the degenera- tive changes in the cervical spine based on lumbar disc degeneration through a retrospective review of positional magnetic resonance imaging (MRI) scans.

\section{Materials and Methods}

This study included 152 patients (81 men and 71 women; age, 20-62 years; mean age, 46.9 years) with symptoms related to cervical and lumbar spondylosis with or without a neurogenic component and who had not undergone prior spinal surgery.

This study was approved by the Institutional Review Board at University of Southern California Health Science Campus (IRB protocol no., HS-1400397).

\section{Magnetic resonance imaging technique}

All patients underwent positional cervical and lumbar MRI with a weight-bearing position on the same day. MRI was performed on a 0.6-T MRI scanner (Upright MultiPosition; Fonar Corp., New York, NY, USA) using a flexible surface coil. The imaging protocol included sagittal T1-weighted spin-echo sequences (repetition time [TR]/ echo time [TE], 671/17 ms; slice thickness, $3.0 \mathrm{~mm}$; field of view, $24 \mathrm{~cm}$; matrix, 256×200; and number of excitations [NEX], 2) and T2-weighted fast spin-echo sequences (TR/TE, 3,432/160 ms; slice thickness, $3.0 \mathrm{~mm}$; field of view, $24 \mathrm{~cm}$; and NEX, 2). All sequences were acquired without fat saturation.

\section{Assessment of intervertebral disc degeneration}

The patients' IDD was evaluated using a previously reported modified classification system based on the degenerative changes in the functional spinal unit [8-10]. The classification system was used to establish an IDD grade, from grade 1, indicative of minimal evidence of IDD, to grade 5 , indicative of substantive evidence of IDD. T2-weighted sagittal images of 1,520 intervertebral discs obtained from all the patients in this study were evaluated and classified using the five-grade system. The following intervertebral discs were evaluated: C2-3, C3-4, C4-5, C5-6, and C6-7 for the cervical spine and L1-2, L2-3, L3-4, L4-5, and L5-S for the lumbar spine. Patients were divided into two groups based on the IDD grade for each lumbar segment: normal (grades 1 and 2) and degenerative (grades 3-5) intervertebral discs. Three independent observers were used 
Table 1. The grading system for intervertebral disc degeneration

\begin{tabular}{|c|c|c|c|c|}
\hline Grade & Structure & Signal intensity & Disc height & Structure of FSU \\
\hline I & Homogeneous, bright white & Hyperintense & Normal & Without DH \\
\hline$\|$ & Inhomogeneous with/without horizontal bands & Hyperintense & Normal & Without DH \\
\hline III & Inhomogeneous, gray & Intermediate & Normal to slight decrease & With/without DH \\
\hline IV & Inhomogeneous, gray to black & Intermediate to hypointense & Decreased & With DH/osteophyte \\
\hline V & Inhomogeneous, black & Hypointense & Collapsed & With DH/osteophyte \\
\hline
\end{tabular}

FSU, functional spinal unit; $\mathrm{DH}$, disc herniation.

Table 2. The average grade of cervical intervertebral discs and DDS for cervical spine

\begin{tabular}{ccccccc} 
& C2-3 & C3-4 & C4-5 & C5-6 & C6-7 & DDS \\
Grade & $2.53 \pm 0.59$ & $2.74 \pm 0.55$ & $2.88 \pm 0.6$ & $3.07 \pm 0.64$ & $2.91 \pm 0.7$ & $14.11 \pm 2.53$ \\
\hline
\end{tabular}

Values are presented as mean \pm standard deviation.

DDS, degenerative disc score.

Table 3. The average grade of lumbar intervertebral discs and DDS for lumbar spine

\begin{tabular}{ccccccc} 
& L1-2 & L2-3 & L3-4 & L4-5 & L5-S & DDS \\
Grade & $2.23 \pm 0.67$ & $2.36 \pm 0.68$ & $2.64 \pm 0.71$ & $2.95 \pm 0.75$ & $3.14 \pm 0.86$ & $13.32 \pm 2.62$ \\
\hline
\end{tabular}

Values are presented as mean \pm standard deviation.

DDS, degenerative disc score.

to grade IDD, and their grades were compared to obtain the level of agreement. The grades attributed to each of the five intervertebral discs at the cervical and lumbar spine were summed to provide the respective degenerative disc score (DDS).

\section{Statistical analysis}

The computer software package StatMate III (ATMS Co. Ltd, Tokyo, Japan) was used. Pearson's correlation coefficient and Mann-Whitney $U$-test were used for statistical analysis. A $p$-value of $<0.05$ was considered statistically significant. Kappa $(\kappa)$ value was calculated as a measure of the inter-observer reliability for IDD grading.

\section{Results}

A high level of agreement $(\kappa=0.674)$ was noted among three independent observers who graded IDD. The distribution of IDD grades is reported in Table 1. The average disc grades for each segment and DDSs for the cervical and lumbar spine are shown in Tables 2 and 3, respectively. The highest IDD grade in the cervical spine was identified at C5-6, followed by C6-7 and C4-5 segments. The

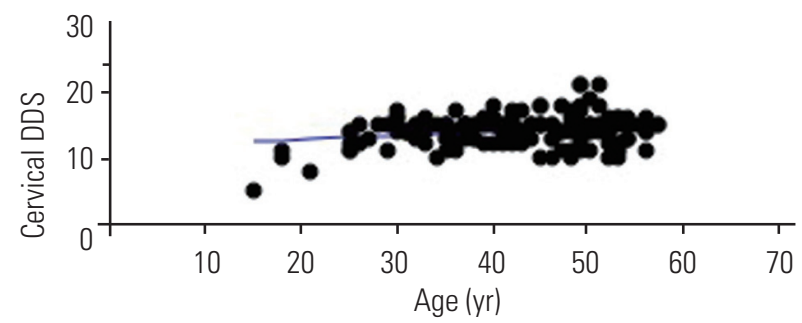

Fig. 1. A significant correlation between age and DDS for the cervical spine; Pearson's correlation coefficient $\left(p<0.01, r=0.25, r^{2}=0.06\right)$. DDS, disc degenerative score.

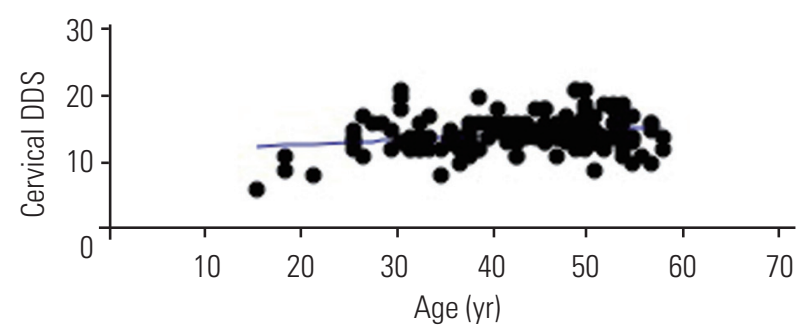

Fig. 2. A significant correlation between age and DDS for the lumbar spine; Pearson's correlation coefficient $\left(p<0.01, r=0.25, r^{2}=0.06\right)$. DDS, disc degenerative score.

IDD grade in the lumbar spine progressively increased from a minimum at the L1-2 segment to more extensive evidence of degeneration in the lower lumbar segments. 
There were positive correlations between age and DDSs (cervical spine: $p<0.01, r=0.25, r^{2}=0.06$; Fig. 1; lumbar spine: $p<0.01, r=0.25, r^{2}=0.06$; Fig. 2 ). In addition, DDSs for the cervical and lumbar spine were positively correlated ( $\left.p<0.001, r=0.44, r^{2}=0.2\right)$ (Fig. 3 ).

The average cervical DDSs for each lumbar segment are shown in Table 4 . We observed significant differences in cervical DDSs between the groups with normal and degenerative intervertebral disc in all lumbar segments. Although there were no significant differences in cervical DDSs in the degenerative lumbar segments, the cervical DDSs at the L1-2 and L2-3 segments tended to be higher than those at the L3-4, L4-5, and L5-S degenerative segments.

\section{Discussion}

The range of motion among the different spinal segments has largely been characterized based on functional plain radiographs obtained in non-weight-bearing positions. This provides evidence of a progressive, cephalad-tocaudal increase in the segmental range of motion, with greater magnitudes of motion in the lower segments of the lumbar spine, L4-5 and L5-S, compared with the upper lumbar segments $[12,13]$. Weight-bearing alters the biomechanical state of intervertebral discs, with reported

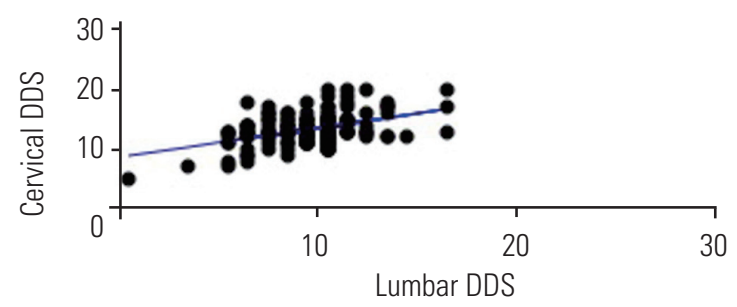

Fig. 3. A significant correlation between DDSs for the cervical and lumbar spine; Pearson's correlation coefficient $(p<0.001, r=0.44$, $r^{2}=0.2$. DDS, disc degenerative score.
$5 \%-15 \%$ decrease in the height and volume of lumbar spine discs in the weight-bearing compared with the non-weight-bearing positions [14,15]. Therefore, imaging studies using non-weight-bearing positions may not accurately reflect the functional kinematics of the human spine. Using positional MRI to investigate the effects of degenerative changes in the lumbar intervertebral discs on the kinematics of the lumbar spine, Lee et al. $[16,17]$ reported a significantly larger range of motion at the L2-3, L3-4, and L4-5 segments compared with the L1-2 and L5-S segments $[11,16,17]$. Our results demonstrated that degenerative changes in the lumbar intervertebral discs are largest in the lower lumbar segments (L4-5 and L5-S segments), with lower evidence of degenerative changes in the upper lumbar segments (L1-2 and L2-3 segments). This lack of concordance between the range of segmental motion, weight-bearing disc volume, and IDD grade may be explained by the findings of Nachemson et al. [18] that the degree of degenerative changes in the lumbar segments does not correlate with the segmental range of motion. The progressive increase in degenerative changes from the upper to the lower segments of the lumbar spine agrees with our predicted pattern of IDD based on the weight-bearing function of the lumbar spine. In particular, the lower lumbar spine is the mechanical fulcrum between the spine and the pelvis. Therefore, the lower lumbar segments might be subjected to the highest magnitudes of mechanical stress during activities of daily living and, as a result, would also be the most influenced by somatotopic, genetic, and environmental factors.

In our study, age reflected the grade of degenerative changes in the cervical and lumbar spine, and there was a significant positive association of the degenerative changes between the cervical and lumbar spine. In particular, we found that participants with degenerative changes in the upper segments of the lumbar spine (L1-2 or L2-3

Table 4. The average DDSs for cervical spine in each lumbar segment

\begin{tabular}{lccccc} 
& L1-2 & L2-3 & L3-4 & L4-5 & L5-S \\
Normal disc & $13.82 \pm 2.32(111)$ & $13.67 \pm 2.23(97)$ & $13.67 \pm 2.51(67)$ & $13.05 \pm 2.46(39)$ & $13.06 \pm 2.42(32)$ \\
Degenerative disc & $14.9 \pm 2.26(41)$ & $14.89 \pm 2.36(55)$ & $14.46 \pm 2.16(85)$ & $14.48 \pm 2.2(113)$ & $14.39 \pm 2.25(120)$ \\
Significant difference & $<0.05^{*}$ & $<0.01^{* *}$ & $<0.05^{*}$ & $<0.01^{* *}$ & $<0.05^{*}$ \\
\hline
\end{tabular}

Values are presented as mean \pm standard deviation (number of subjects). Mann-Whitney $U$-test: compared with normal and degenerative disc groups.

DDS, degenerative disc score.

" $p<0.05$, ${ }^{* *} p<0.01$. 
segments) were more likely to have a higher DDS of the cervical spine. Based on our study results, we propose the need to consider the presence of cervical spine disorders in patients being evaluated and treated for lumbar spine disorders. A prognostic determination of degenerative changes in the cervical spine based on the assessment of IDD of the spinal segments in the upper lumbar spine could lower the incidence of missed diagnosis of cervical spine disorders in patients presenting with lumbar spine symptomology.

The limitations of our study need to be considered in the application of its outcome to practice. Foremost, only IDD in the cervical and lumbar spine was assessed, without consideration of neurological symptoms and impairments, such as the degree of spinal cord or cauda equina entrapment. Therefore, our results proof-of-principle data to support the development of a larger, population-based study to establish the prevalence of degenerative changes for all segments of the spine, as well as to clarify the relationships between the degenerative changes at multiple spinal levels.

\section{Conclusions}

Our study showed that patients with degenerative changes in the upper lumbar segments are more likely to have certain degree of cervical spondylosis. This information could lower the incidence of missed diagnosis of cervical spine disorders in patients presenting with lumbar spine symptomology.

\section{Conflict of Interest}

No potential conflict of interest relevant to this article was reported.

Disclosures outside of submitted work: ZB-Xenco Medical (consultancy), AO Spine (consultancy); JCW-Royalties-Biomet, Seaspine, Amedica, Synthes; investments/ options: Bone Biologics, Expanding Ortho, Pearldiver, Flexuspine, Fziomed, Benvenue, Promethean, Nexgen, Electrocore, Surgitech; board of directors: North American Spine Society, AO Foundation, Cervical Spine Research Society; editorial boards: Spine, JAAOS, The Spine Journal, Clinical Spine Surgery, Global Spine Journal; and fellowship funding (paid to institution): AO Foundation.

\section{Acknowledgments}

The study was supported by departmental funds. Authors would like to thank AiM Radiology Medical Group, especially to Yusuf A. Khan, Sameer U. Khan and Aziza Qadir MD for their help on obtaining and uploading kMRI images into the database.

\section{References}

1. Zigler JE, Strausser DW. The aging spine. In: Fardon DF, Garfin SR, editors. Orthopaedic knowledge update: spine 2. Rosemont (IL): American Academy of Orthopaedic Surgeons; 2002. p.123-33.

2. Avinash GP, Ioannis NG, Leonald IV. Biomechanics of the spine. In: Spivak JM, Connolly PJ, editors. Orthopaedic knowledge update: spine 3. Rosemont (IL): American Academy of Orthopaedic Surgeons; 2006. p.25-32.

3. Hanaei S, Abdollahzade S, Khoshnevisan A, Kepler CK, Rezaei N. Genetic aspects of intervertebral disc degeneration. Rev Neurosci 2015;26:581-606.

4. Eskola PJ, Mannikko M, Samartzis D, Karppinen J. Genome-wide association studies of lumbar disc degeneration: are we there yet? Spine J 2014;14:479-82.

5. Nakki A, Battie MC, Kaprio J. Genetics of disc-related disorders: current findings and lessons from other complex diseases. Eur Spine J 2014;23 Suppl 3:S35463.

6. Battie MC, Lazary A, Fairbank J, et al. Disc degeneration-related clinical phenotypes. Eur Spine J 2014;23 Suppl 3:S305-14.

7. Ikegawa $S$. The genetics of common degenerative skeletal disorders: osteoarthritis and degenerative disc disease. Annu Rev Genomics Hum Genet 2013;14:245-56.

8. Pfirrmann CW, Metzdorf A, Zanetti M, Hodler J, Boos N. Magnetic resonance classification of lumbar intervertebral disc degeneration. Spine (Phila $\mathrm{Pa}$ 1976) 2001;26:1873-8.

9. Miyazaki M, Hong SW, Yoon SH, et al. Kinematic analysis of the relationship between the grade of disc degeneration and motion unit of the cervical spine. Spine (Phila Pa 1976) 2008;33:187-93.

10. Morishita Y, Hida S, Miyazaki M, et al. The effects of the degenerative changes in the functional spinal unit on the kinematics of the cervical spine. Spine (Phila 
Pa 1976) 2008;33:E178-82.

11. Tan Y, Aghdasi BG, Montgomery SR, Inoue H, Lu C, Wang JC. Kinetic magnetic resonance imaging analysis of lumbar segmental mobility in patients without significant spondylosis. Eur Spine J 2012;21:2673-9.

12. Hayes MA, Howard TC, Gruel CR, Kopta JA. Roentgenographic evaluation of lumbar spine flexionextension in asymptomatic individuals. Spine (Phila Pa 1976) 1989;14:327-31.

13. Pearcy M, Portek I, Shepherd J. Three-dimensional $\mathrm{x}$-ray analysis of normal movement in the lumbar spine. Spine (Phila Pa 1976) 1984;9:294-7.

14. Malko JA, Hutton WC, Fajman WA. An in vivo magnetic resonance imaging study of changes in the volume (and fluid content) of the lumbar intervertebral discs during a simulated diurnal load cycle. Spine (Phila Pa 1976) 1999;24:1015-22.
15. Botsford DJ, Esses SI, Ogilvie-Harris DJ. In vivo diurnal variation in intervertebral disc volume and morphology. Spine (Phila Pa 1976) 1994;19:935-40.

16. Lee SH, Daffner SD, Wang JC, Davis BC, Alanay A, Kim JS. The change of whole lumbar segmental motion according to the mobility of degenerated disc in the lower lumbar spine: a kinetic MRI study. Eur Spine J 2015;24:1893-900.

17. Lee SH, Daffner SD, Wang JC. Does lumbar disk degeneration increase segmental mobility in vivo?: segmental motion analysis of the whole lumbar spine using kinetic MRI. J Spinal Disord Tech 2014;27:1116.

18. Nachemson AL, Schultz AB, Berkson MH. Mechanical properties of human lumbar spine motion segments: influence of age, sex, disc level, and degeneration. Spine (Phila Pa 1976) 1979;4:1-8. 